\title{
Las acciones de comunicación, la web 2.0 y la actividad promocional
}

\author{
Javier SENDRA GARCÍA \\ Universidad Complutense de Madrid \\ Javier.sendra@pepsico.com \\ María Tejerina ArReal \\ Universidad de Murcia \\ tejerina@um.es \\ Ma Luisa García GuARDia \\ Universidad Complutense de Madrid \\ mluisagarcia@ccinf.ucm.es
}

\section{Resumen:}

La presente investigación aborda un área de conocimiento poco estudiado en el ámbito académico: las acciones de comunicación, la web 2.0 y la actividad promocional. Si bien es cierto que todas estas áreas han sido estudiadas por separado, no lo han sido tanto respecto a la interacción que se produce entre ellas, ni desde una perspectiva de globalidad.

Palabras clave: Web 2.0; comunicación; actividad promocional; consumidor.

\section{Comunication actions, web 2.0 and promotional activity}

\begin{abstract}
:
This research addresses an understudied area of knowledge in Academia: the communication actions, Web 2.0 and promotional activities. All these areas have been widely studied separately, but the relationships between them has not been addressed so extensively and from a global perspective.
\end{abstract}

Key Words: Web 2.0; communication; promotional activity; consumer.

\section{Referencia normalizada:}

Sendra García, J., Tejerina Arreal, M. y García Guardia, M. L. (2014): Las acciones de comunicación, la web 2.0 y la actividad promocional. Historia y Comunicación Social. Vol. 19. Núm. Especial Marzo. Págs. 439-454.

Sumario: 1. Introducción. 2. La Actividad Promocional: marco, aplicaciones y evolución. 2.1. La comunicación al consumidor. 2.2. Objetivo de la promoción de ventas. 2.3. Beneficios de la promoción para el comprador. 2.4. Herramientas promocionales. 3. Objetivos. 4. Metodología. 5. Conclusiones. 6. Bibliografía. 


\section{Introducción}

En los últimos años se ha producido una transformación en el ámbito del marketing, siendo destacable la aparición del llamado Marketing digital e Interactivo. En este contexto de cambios constantes, el consumidor se ha convertido en una pieza clave por la capacidad de interacción en el entorno de la comunicación persuasiva. Por ello, los especialistas en mercadotecnia se han interesado por obtener directamente información sobre el producto para poder influir sobre el comportamiento de compra o, realizar algo tan valioso, como convertirse en prescriptores de los mismos.

Actualmente, el objetivo de las marcas se basa en llegar a un consumidor cada vez más disperso entre una multitud de medios publicitarios lo que ha propiciado la necesidad de utilizar nuevos sistemas de medición de la información ofrecida a través de Internet así como de las nuevas formas de comunicación que están teniendo lugar. Estos sistemas se centran en la segmentación de los mensajes en función del tipo de consumidor teniendo en cuenta dónde y cuándo adopta sus decisiones de compra permitiendo, de esta manera, conocer la atención de la audiencia y calificarla.

Por otro lado, el actual entorno económico unido a la competencia existente entre los diversos medios existentes hace que cualquier inversión publicitaria por parte de los fabricantes esté orientada a proporcionarles mejores resultados a corto plazo, lo que hace que esta inversión se encuentre sujeta a mayores controles de rentabilidad, para lo que resulta imprescindible que el proceso inversor publicitario sea medible, dos características que reúne la actividad promocional (Fernández Varona, Llorente Barroso, García Guardia, 2012).

De entre las TIC, y más concretamente Internet, la web 2.0 ha propiciado el desarrollo de estrategias empresariales orientadas hacia la importancia de la gestión de contenidos. La empresa se enfrenta desde hace "relativamente" poco tiempo a un contacto bidireccional: escuchar, conversar y relacionarse.

Actualmente, la relación entre el producto o marca y el consumidor pasa por una relación abierta que se desarrolla en frentes variados, fruto de la combinación de las relaciones tradicionales con las nuevas relaciones producidas a través de las TIC, produciéndose una interacción constante entre ambas formas de relación.

En esta realidad en permanente trasformación se hace pertinente la presente investigación, que tiene como objetivo la influencia de la web 2.0 en el conocimiento del consumidor de un producto promocionado. 


\section{La actividad promocional: marco, aplicaciones y evolución}

\subsection{Objetivo de la promoción de ventas}

Reinares y Calvo (1999) apuntan que el objetivo principal de la promoción no debería ser el incremento de ventas y de cuota de mercado, que es sólo un objetivo secundario, sino que debería haber detrás unos objetivos primarios relacionados con reglas del marketing que pasa por conocer bien al cliente y disponer de una buena tipología de clientes a los que dirigir diferencialmente la publicidad y las acciones de comunicación sobre los productos o servicios de la empresa con el objetivo de su fidelización.

Por las razones apuntadas, es necesario integrar el punto de vista comercial (táctico), con el de la marca (estratégico). Si bien este enfoque puede adolecer de ser demasiado teórico, se considera que es importante que el planificador tenga una visión estratégica del plan promocional anual, sin perder de vista los objetivos de ventas que son la principal fuente de ingresos de cualquier compañía de GC (Gran Consumo), al generar el beneficio suficiente para poder reinvertir parte de la plusvalía en nuevas acciones de publicidad y promociones que aseguren la creación y desarrollo del Valor de Marca, y que es a la postre el mejor garante de las ventas en el futuro. En algunas ocasiones, el planificador tendrá que elegir entre uno de los dos enfoques a la hora de canjear imagen de marca por menor ventas, o viceversa.

Este enfoque es defendido por autores como Davis (1992), que argumenta que el verdadero efecto de la promoción de ventas sólo puede alcanzarse si la actividad está coordinada con las otras actividades del mix comunicacional, y esto requiere buena planificación. En particular, la naturaleza complementaria de la promoción de ventas y la publicidad debería explotarse a través del uso de mensajes y temas comunes, la coincidencia en el tiempo y en el target, y la designación de recursos, específicamente los presupuestos.

En esta línea, Fill (1995) argumenta que las promociones de venta planificadas como una secuencia de actividades predeterminada reflejan las necesidades de comunicación de una marca en el largo plazo, y tienen mayor probabilidad de tener éxito que aquellas que son simples reacciones a las acciones de la competencia o a la marcha del mercado.

Se coincide por lo tanto, con la necesidad de tener una visión estratégica para la marca en el medio y largo plazo, con respecto a su posicionamiento y al uso de los distintos elementos del marketing mix que confieran un programa de progresión para la marca acorde con los parámetros establecidos en la conocida matriz del BCG (Boston Consulting Group). Esta visión estratégica debe establecer unos objetivos de la promoción de ventas claros y definidos que permitan comprobar posteriormente su grado de cumplimiento y alcance (tabla 1). Estos objetivos se pueden desglosar en estratégicos y tácticos. Los estratégicos deben ser acordes con el plan de marketing de la marca o marcas involucradas y ser consistentes con su posicionamiento 
(desarrollada por Reinares y Calvo (1999). Esta clasificación comprende cuatro tipos: convertir, atraer, retener y aumentar objetivos que se intentarán cumplir mediante las distintas aplicaciones tácticas dirigidas, bien al consumidor final, bien al canal de distribución. Se adjunta un cuadro explicativo de la relación entre objetivos tácticos y aplicados.

Tabla 1. Objetivos tácticos y objetivos aplicados a la promoción de venta

\begin{tabular}{|c|c|}
\hline $\begin{array}{c}\text { Objetivos } \\
\text { Tácticos }\end{array}$ & Objetivos Aplicados \\
\hline $\begin{array}{l}\text { Convertir: } \\
\text { Dirigido a } \\
\text { consumidores } \\
\text { de la } \\
\text { competencia }\end{array}$ & $\begin{array}{l}\text { Potenciar el conocimiento del producto entre usuarios de otras marcas } \\
\text { Incentivas la prueba entre usuarios de otras marcas } \\
\text { Elevar el nivel de interés de los usuarios actuales } \\
\text { Incentivar a los intermediarios a comercializar un producto en periodo de } \\
\text { relanzamiento } \\
\text { Lograr mejor exhibición en el punto de venta }\end{array}$ \\
\hline $\begin{array}{l}\text { Atraer: } \\
\text { Dirigido a } \\
\text { consumidores } \\
\text { de otra } \\
\text { categoría }\end{array}$ & $\begin{array}{l}\text { Potenciar el conocimiento } \\
\text { Incentivar la prueba } \\
\text { Elevar el nivel de interés de los no usuarios en el producto } \\
\text { Incentivar a los intermediarios que permiten llegar a los nuevos segmentos o } \\
\text { mercados. } \\
\text { Lograr mejor exhibición en el punto de venta del canal de intermediarios }\end{array}$ \\
\hline $\begin{array}{l}\text { Retener: } \\
\text { Dirigido a } \\
\text { mantener los } \\
\text { consumidores } \\
\text { actuales }\end{array}$ & $\begin{array}{l}\text { Consolidar la lealtad del comprador hacia la marca } \\
\text { Potenciar la frecuencia y la cantidad comprada ( rotación) } \\
\text { Regularizar la demanda del producto } \\
\text { Evitar los efectos negativos de un incremento del precio } \\
\text { Aminorar los efectos negativos de promociones de competidores }\end{array}$ \\
\hline $\begin{array}{l}\text { Aumentar: } \\
\text { Dirigido a } \\
\text { fidelizar los } \\
\text { consumidores } \\
\text { actuales }\end{array}$ & $\begin{array}{l}\text { Incentivar la prueba en nuevas aplicaciones o momentos de consumo } \\
\text { Consolidar la lealtad hacia la marca } \\
\text { Potenciar la frecuencia y la cantidad comprada ( rotación) } \\
\text { Regularizar la demanda } \\
\text { Neutralizar un aumento de precio }\end{array}$ \\
\hline
\end{tabular}

Fuente: Adaptado de Reinares y Calvo (1999)

Una forma de integrar conceptualmente los objetivos anteriores es resumiendo las dos coordenadas del comportamiento que intenta modificar o alterar la promoción:

- Convertir y atraer:

- La penetración, entendida como la variación de la actitud del comprador en su relación con la marca: haciendo que el comprador habitual compre más e incorporando al comprador no habitual.

- Retener y aumentar:

- La frecuencia de consumo, ya que al incrementarse el ratio de consumo se acorta el periodo de espera hasta la próxima compra.

Finalmente, lo ideal para el fabricante es que la promoción no sólo incremente el ratio de compra del comprador por su implicación en la cuota del presupuesto del hogar, sino que lo verdaderamente beneficioso es que aumente el ratio de consumo de 
ese individuo comprador. Esto, como comprobaremos, va a depender de la categoría objeto de estudio que limitará la eficacia de la actividad promocional de una marca, tanto por la sensibilidad que exista a las promociones (ratio de compra), como por la propensión al consumo de un producto de una categoría por el hecho de tener más cantidad almacenada en el hogar (ratio de consumo).

En caso de que el incremento de las ventas tenga un origen en la aceleración de la compra, sin un reflejo en el aumento del consumo; lo único que ha ocurrido es un desplazamiento de la compra, circunstancia muy habitual en productos de gran consumo.

El fabricante tiene que ser realista a la hora de plantearse una promoción, sobre todo, entender que se puede lograr con ella, y lo que no; en este último caso habría que pensar en otra herramienta para conseguir el objetivo deseado.

En la siguiente tabla se apuntan los beneficios utilitarios y hedonistas del comprador frente a la promoción de ventas.

Tabla 2. Beneficios potenciales del comprador

\begin{tabular}{|l|l|}
\hline \multicolumn{1}{|c|}{ Efecto } & \multicolumn{1}{c|}{ Beneficio } \\
\hline Ahorro & Reducción del precio o desvío del precio de referencia. \\
\hline Calidad & $\begin{array}{l}\text { Reducción del precio o formato alternativo. Puede provocar que se compre } \\
\text { un producto de una mayor calidad percibida (accesibilidad), gastando más } \\
\text { dinero. }\end{array}$ \\
\hline Conveniencia & $\begin{array}{l}\text { Reducción del coste de tiempo de búsqueda, y facilita la toma de decisiones } \\
\text { (exposición adicional). }\end{array}$ \\
\hline $\begin{array}{l}\text { Expresión de } \\
\text { Valor }\end{array}$ & $\begin{array}{l}\text { Cubre objetivos personales o éticos del comprador, que le gusta ser visto } \\
\text { como comprador responsable y avispado. }\end{array}$ \\
\hline Exploración & $\begin{array}{l}\text { La dinámica promocional cambiante provoca el interés y la necesidad } \\
\text { intrínseca de explorar; alternar marcas y buscar información ( Kahn 1995.) }\end{array}$ \\
\hline Entretenimiento & Algunas promociones son divertidas de participar y observar. \\
\hline
\end{tabular}

Fuente: Chandon et al (2000)

Como se señaló anteriormente, habrá que plantearse las herramientas necesarias para conseguir estos objetivos potencias, que pueden ser la publicidad u otros elementos de la comunicación, así como también otros componentes del marketing mix.

\subsection{Beneficios de la promoción para el comprador}

En este escenario es muy revelador el trabajo de Chandon et al (2000), que configuran un marco de congruencia entre la marca y el tipo de promoción a utilizar, dividiendo las marcas en dos tipos: las de valor utilitario y las de valor hedonista. A cada una de ellas le conviene un tipo de promoción distinta, según el beneficio que proporcionan al comprador. 
Los tres primeros beneficios los consideran utilitarios; y los dos últimos, hedonistas, mientras que el beneficio de "expresión de valor" se considera de naturaleza mixta.

Las conclusiones de su trabajo son relevantes:

- Las promociones de valor promocionan más beneficios hedonistas y utilitarios que las promociones monetarias.

- Todos los beneficios, a excepción de la calidad, contribuyen a la valoración de promociones monetarias y no monetarias (de valor).

- Para las marcas de alto valor; las promociones de ventas son más eficaces cuando proporcionan beneficios congruentes con aquellos que manifiestan la marca promocionada. Específicamente, las promociones son más eficaces para las marcas utilitarias que para las marcas hedonistas.

- Del mismo modo las promociones de valor son relativamente más eficaces en los productos hedonistas, que para los utilitarios.

- Estas conclusiones se consideran relevantes para la gestión promocional de las marcas, que debe ser acorde con su posicionamiento en la categoría, lo que nos da una idea de la utilización de la promoción de ventas como instrumento de creación de valor de Marca, o al menos, de no erosión del mismo.

\subsection{Herramientas promocionales}

Los profesionales del sector de GC no se ponen de acuerdo en la definición conceptual de promoción de ventas. Existe una corriente de pensamiento que sostiene que herramientas como los folletos o la exposición adicional y degustaciones no son promociones, ya que no añaden valor, al tratarse de meros soportes para comunicar el estímulo de una promoción (reducción PVP, regalos, etc.), que es lo que el comprador percibe al ver la cabecera o el folleto.

Sin embargo, la exposición adicional aumenta la venta por dos motivos:

- El comprador "encuentra" el producto fácilmente, se lo ponen "a mano" (Ahorro de tiempo).

- Inconscientemente asocia espacio adicional con oferta de precio (Ahorro económico)

En función de ello se puede considerar la promoción de ventas como cualquier estímulo por el cual el minorista o el fabricante intentan influenciar el comportamiento del comprador en el acto de compra, sobre todo en lo que a los productos de gran consumo se refiere. Es por lo tanto, un medio para conseguir un fin que es la venta por impulso.

Esta definición coincide con la interpretación que hace Chandon et al (2000) al referirse a los distintos beneficios potenciales de la promoción de ventas para el 
comprador, entre los que figuran el ahorro (económico), y la conveniencia (ahorro de tiempo).

Otro argumento para considerar exposición y folletos como acciones promocionales es la posibilidad de su medición o seguimiento, ya que los datos de los institutos proveedores de información (Nielsen, IRI, etc.) incluyen estos dos elementos en sus informes.

En cuanto a los elementos que Reinares y Calvo (1999) seleccionan como paradigmáticos, con el objetivo de conseguir un instrumento de comunicación único, nos quedamos con uno de ellos como realmente indispensable: cualquier promoción está limitada en el tiempo, ya que de otro modo se convertiría en un componente indiferenciado con respecto a otras campañas que se puedan realizar del producto en cuestión.

Reinares y Calvo (1999) consideran la exposición adicional (cabecera, chimenea, isla), como un elemento de merchandising englobado dentro de la publicidad en el punto de venta, es decir, como un soporte donde se publicita una promoción de ventas, pero no intrínsecamente una promoción. Dentro de esta concepción el estímulo se puede proyectar en diversos soportes, pero la promoción no es el soporte, sino el estímulo.

Sin embargo, la exposición adicional es una actividad promocional, ya que en muchos casos no existe el estímulo que da contenido al soporte (por ejemplo cabecera sin reducción de precio), y el soporte es la promoción en sí, cuyo objetivo principal es provocar la venta por impulso añadiendo valor al producto al hacerlo accesible y suponer un ahorro de tiempo en la búsqueda de la marca; siendo un hecho comprobado que la percepción del comprador con respecto a dicha exposición es que está asociada a una reducción de precio. Se dan en este caso dos tipos de ahorro; por un lado el económico (percibido); y por otro lado de tiempo.

Las herramientas más importantes utilizadas en los productos de Gran Consumo, atendiendo a nuestro criterio de considerar la promoción de venta como un conjunto de incentivos del acto de compra, son las siguientes:

- Folletos.

- Exposición adicional o "display" (Cabecera, chimenea, pilada e isla.)

- Regalo Directo (“on pack”; o de entrega en caja).

- Sorteo (viaje, premio).

- Competición (requiere habilidad).

- Reducción temporal del precio.

- Promoción más cantidad $(2 \times 1)$.

- Degustaciones punto de venta.

- Puntos extra del programa de fidelización. 
- Cupones descuento.

- Anuncio TV o prensa de comunicación de PVP.

La mayoría de ellos se ejecutan en el punto de venta, pero algunos, como el folleto, se envían a los hogares. Lo mismo sucede con los anuncios de prensa o en promociones específicas de TV en las que aparece el precio de uno o varios productos en oferta como reclamo para atraer al comprador a la tienda.

Otra modalidad promocional son las llamadas "cabeceras de góndola" en los extremos de los lineales, se consideran zonas calientes dentro de la tienda y son objeto de planificación anual por parte del distribuidor que genera ingresos sustanciales alquilando el espacio a diversos fabricantes. En ocasiones estas aportaciones de los fabricantes son objeto de discusión y negociación en la firma de las plantillas comerciales anuales.

De las herramientas anteriormente citadas habría que distinguir entre las ligadas a una marca o producto concreto, y las promociones de ambiente (por ejemplo, días especiales) o de establecimientos (por ejemplo, aniversarios de la cadena). En estos casos, la promoción tiene un efecto paraguas que alberga diversas marcas y categorías incluyendo decoración especial y animación en el punto de venta.

\section{Objetivos}

- Describir las relaciones existentes entre las acciones de comunicación, la Web 2.0. y la actividad promociones.

- Analizar las nuevas formas de comunicación de la actividad promocional con la aparición del Marketing digital e interactivo.

- Estudiar los nuevos sistemas de medición de la información ofrecida a través de Internet.

\section{Metodología}

Una red social (SRS) es una estructura social que se puede representar en forma de uno o varios grafos en el cual los nodos representan individuos (a veces denominados actores) y las relaciones que se establecen entre ellos (www.wikipedia.es).

La notoriedad alcanzada por las redes sociales ha venido unida al incremento de internautas que han decidido formar parte de ellas.

$\mathrm{Su}$ origen de las redes data del 1995, fecha en que Randy Conrads puso en marcha www.classmates.com. La primera red, tal como hoy la entendemos, tuvo su origen en 
1997 y se llamaba SixDegrees.com, la cual permitía a los usuarios crear perfiles, lista de amigos y amigos de sus amigos.

De 1997 a 2001, Asian Avenue, Blackplanet y MiGente permitían a los usuarios que se identificaran, estableciendo relaciones personales o profesionales, que crearan perfiles y que identificaran amigos en la red sin pedir la aceptación por parte de estos. Con el tiempo, algunas de estas redes han desaparecido, siendo en la actualidad las más visitadas: Facebook, MySpace, LinkedIn, Twitter, etc. Funcionan con millones de internautas que las visitan diariamente.

Desde un punto de vista empresarial, las redes sociales de relevancia son aquellas que tienen proyección comercial y profesional. Su fuerza está en ser una red de apoyo a los consumidores en dicho entorno profesional, siendo una herramienta para buscar trabajo, conectar empresas y potenciar comunidades virtuales ligadas a éstas. LinkedIn es la red que representa la filosofía empresarial tal vez más conocida.

MySpace es una red que permite a los usuarios personalizar sus páginas de forma más eficaz, y esta es la característica que la diferencia de otras. Su origen estuvo ligado a un público adolescente, existiendo en la actualidad tres perfiles de usuarios: músicos/artistas, jóvenes y adultos.

Facebook nació ligada a las redes universitarias, siendo 2004 el momento en que se obligó a los usuarios a proporcionar los correos electrónicos. Esto convirtió a la red en un espacio relativamente cerrado. Con posterioridad se ha ido ampliando el perfil de usuarios. Una de las principales cualidades es la capacidad de los desarrolladores externos de crear aplicaciones permitiendo a los visitantes personalizar sus perfiles y realizar múltiples tareas tales como: ser fan, decidir preferencias, etc.

Desde un punto de vista tecnológico, las redes sociales poseen unas aplicaciones comunes (correos electrónicos y protocolos) y tecnologías estándares que han sido parte de su éxito y que han servido como herramienta de comunicación: subir y bajar información (fotos o información sobre el perfil), mensajería instantánea, conexión instantánea uno a uno, alta capacidad de almacenamiento, búsqueda de contactos, diseminación de información personal (nombre, localización geográfica, historial académico, intereses comunes), compartir y etiquetar fotos y vídeos (lo que de alguna manera posibilita personalizar y estructurar la página), creación de texto en tiempo real, etc.

Estas características de las redes sociales ha tenido sus consecuencias en el mundo comercial gracias a todas las nuevas posibilidades desarrolladas en poco tiempo: el fenómeno "hacerse fan de una marca", y todos los cambios que ha supuesto desde el punto de vista de la comunicación, han añadido nuevos significados a las relaciones comerciales: SRS (Safety Respons Service) de una marca determinada con el fin de establecer comunicaciones one to one integradas en campañas de comunicación internas y externas de las compañías; redes sociales de compras, siendo estas últimas un espacio donde los consumidores pueden consultar todo tipo de dudas sobre los productos o servicios; conocer las opiniones de otros consumidores; ser constructo- 
res de contenidos; votar a sus productos favoritos; conocer gente con las mismas aficiones; o comprar con un solo click, a esta tendencia se la denomina Shopping 2.0.

En resumen, nos encontramos ante un universo interactivo que se ha ido desarrollando y volviendo cada vez más complejo, en el que la convergencia de medios está re-escribiendo la manera en que los sujetos se relacionan desde un punto de vista comercial: marketing digital, eCommerce, redes sociales, contenidos de gestión y conectividad.

\section{Imagen 1. Notoriedad de las Redes Sociales}

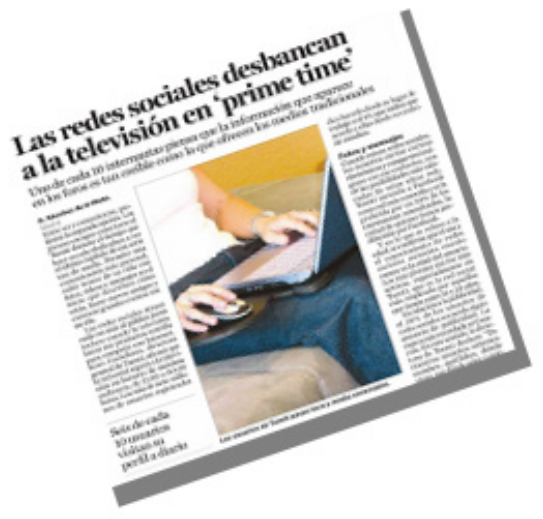

Analizado alguna de las redes sociales se pueden encontrar diferencias significativas entre las mismas en función de su objetivo principal. Por ejemplo, Foursquare es una interesante red social que ha conseguido captar la atención de una buena cantidad de internautas últimamente, en gran parte por su estrategia para que los que se registren se animen a seguir activos en el servicio, a diferencia de Facebook y Twitter cuyo objetivo es simplemente conectar a los usuarios con sus amigos y mantenerse actualizado tanto de lo que ocurra en el mundo como lo que ocurra en su círculo de usuarios.

Siguiendo con el análisis de Foursquare consiste en una red social en la que los usuarios realizan "check-ins" en los locales que visitan mientras comparten recomendaciones y opiniones acerca de dichos locales con sus contactos. Para poder hacerlo de forma efectiva, Foursquare tiene disponibles aplicaciones móviles capaces de usar el GPS (que ya incluyen la mayoría de dispositivos como el Nexus One o el iPhone) del dispositivo para realizar check-ins de la forma más sencilla posible. Dejan, además, libertad al usuario para introducir toda la información de un local en el caso de que nadie de la red haya pasado por allí antes. 
Imagen 2. Características de la red social Foursquare
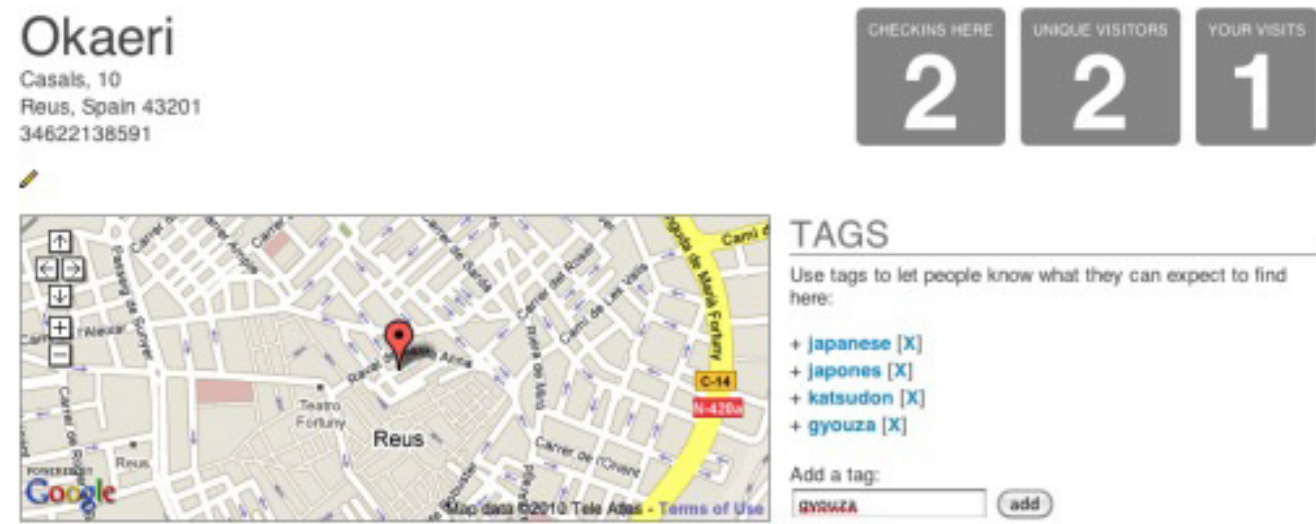

Pero la clave de Foursquare es, sobretodo, que convierte el hecho de ir marcando todos los sitios visitados en un juego. Cada vez que visitas un local (que puede ser un bar, una discoteca, un aeropuerto, un hotel, un museo, una convención...) ganas una cierta cantidad de puntos y, en ocasiones especiales en las que alcanza ciertos requisitos, incluso una medalla. También tenemos algunas bonificaciones extra si entramos en sitios no visitados e introducimos los datos, o si realizamos algún check-in en el extranjero.

Sólo por esto, los usuarios tienen interés en conseguir puntos, de modo que se despierta el instinto competitivo y se consigue un uso intensivo de la red social. Además, el hecho de que Foursquare se base en la localización representa una buena oportunidad para varios frentes. En primer lugar, la red puede mostrar publicidad dependiendo de dónde estamos, un modo selectivo de promocionar productos y servicios que está ganando mucho interés por parte de grandes compañías como Google y Apple, que han adquirido empresas de publicidad móvil (AdMob y Quattro Wireless respectivamente).

Imagen 3. Dispositivos variados de terminales móviles
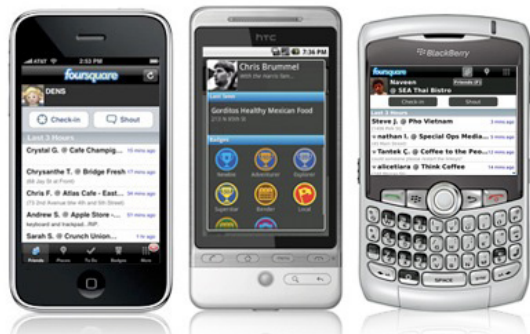

Algo positivo a remarcar de Foursquare es que tiene disponibles aplicaciones móviles para prácticamente cualquier teléfono móvil, ampliando tremendamente el 
abanico de usuarios que pueden participar en el 'juego' de esta red social. Tenemos la aplicación en la App Store para el iPhone, la aplicación en la tienda de Android y una tercera para móviles BlackBerry. Por supuesto, todas gratuitas.

La integración con otras redes como Twitter y Facebook también es un punto fuerte, ya que de este modo todos nuestros contactos se dan cuenta de que estamos usando Foursquare (captando la atención) como de los locales que visitamos y que marcamos.

La investigación realizada compara un conjunto de variables de las denominadas TIC con la importancia de la web 2.0 desde el punto de vista de la eficiencia en la promoción de ventas de los productos de gran consumo. Las variables que se comparan son las siguientes:

- Web-Microsite.

- SMS.

- MMS.

- Digital signage.

- Correo electrónico.

- Redes Sociales.

- Geolocalización-Red Social.

- Códigos Bidi.

- TV Digital.

La metodología seleccionada incluye dos técnicas: Delphi y la relativa al análisis de Redes Sociales, para lo que se ha utilizado la técnica cualitativa de Análisis de Fuentes Documentales. La investigación ha sido de carácter predictivo, mediante un proceso sistemático e iterativo para conseguir la obtención de las opiniones de 68 expertos ( $42,6 \%$ con perfil académico y $57,4 \%$ de tipo profesional). El procedimiento, el Método Delphi, ha permitido el cálculo estadístico descriptivo en medidas de localización (moda, media y mediana), así como, el coeficiente de variación, la variación del rango intercuartílico reducido o la variación del coeficiente de variación.

A continuación se muestran los porcentajes de valoración obtenidos para cada una de las herramientas consideradas. Podemos ver que la puntuación más alta se obtiene para las redes sociales más geolocalización (4,10 de media con un IC al 95\% para la misma entre 3,86 y 4,35$)$ y redes sociales $(4,07$ de media con un IC al $95 \%$ entre 3,87 y 4,28), seguidas de la Web-Microsite (3,53 con un IC al $95 \%$ entre 3,28 y 3,78), del Digital Signage (3,19 con un IC al 95\% entre 2,94 y 3,44), del SMS (telefonía móvil) (3,18 con un IC al $95 \%$ entre 2,91 y 3,44$)$, de la TV Digital $(3,13$ con un IC al $95 \%$ entre 2,91 y 3,35$)$, de los códigos Bidi $(3,07$ con un IC al $95 \%$ entre 2,83 y $3,31)$, del correo electrónico ( 3,03 con un IC al $95 \%$ entre 2,76 y 3,30), y por último del MMS (telefonía móvil con imagen) (3,01 de con un IC al 95\% entre 2,74 y 3,29). 
Por tanto, en todos los casos la valoración de todas las herramientas es alta, siendo especialmente elevadas las de las redes sociales.

Tabla 3. Porcentajes de valoración de las herramientas

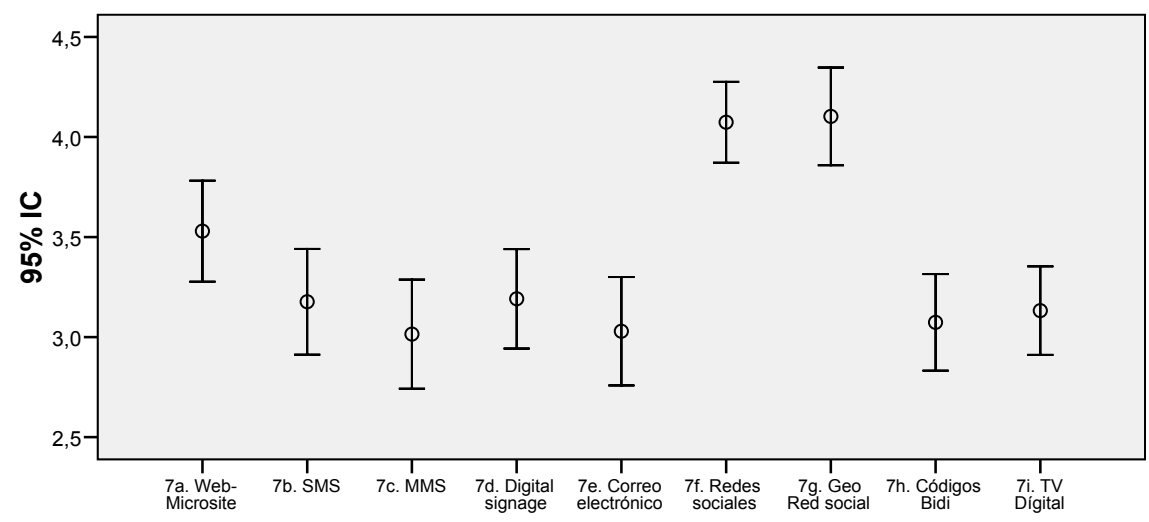

Fuente: elaboración propia

Se muestran los porcentajes de valoración obtenidos para cada una de las herramientas consideradas (tabla 3). Se puede observar que la puntuación más alta se obtiene para las redes sociales más geolocalización (4,10 de media con un IC al $95 \%$ para la misma entre 3,86 y 4,35$)$ y redes sociales $(4,07$ de media con un IC al $95 \%$ entre 3,87 y 4,28), seguidas de la Web-Microsite (3,53 con un IC al 95\% entre 3,28 y 3,78 ), del Digital Signage (3,19 con un IC al $95 \%$ entre 2,94 y 3,44), del SMS (telefonía móvil) (3,18 con un IC al 95\% entre 2,91 y 3,44), de la TV Digital (3,13 con un IC al $95 \%$ entre 2,91 y 3,35$)$, de los códigos Bidi (3,07 con un IC al $95 \%$ entre 2,83 y $3,31)$, del correo electrónico ( 3,03 con un IC al $95 \%$ entre 2,76 y 3,30), y por último del MMS (telefonía móvil con imagen) (3,01 de con un IC al 95\% entre 2,74 y 3,29). Por tanto, en todos los casos la valoración de todas las herramientas es alta, siendo especialmente elevadas las de las redes sociales.

Si se comparan las valoraciones suministradas a cada una de las nueve herramientas consideradas podemos ver que existen diferencias significativas ( $p$-valor $<0,001$ ) entre las valoraciones suministradas aplicando la prueba no paramétrica de Friedman de comparación de muestras emparejadas. Para analizar las fases con valoración diferente del resto se han aplicado pruebas de comparación de dos muestras emparejadas de Wilcoxon para cada par de fases, utilizando la corrección de Bonferroni para comparaciones múltiples en los p-valores obtenidos. Se muestran dos grupos homogéneos de herramientas según su valoración: un primer grupo formado por las redes sociales con o sin geocodificación con valores similares entre sí y significativamente superiores al resto de herramientas, que son similares entre sí. 
Tabla 4. Grado de eficiencia de las redes sociales con geolocalización

Grado de Eficiencia de las Redes Sociales con Geolocalización

\begin{tabular}{|ll|r|r|}
\hline & Frecuencia & Porcentaje \\
\hline Válidos & 1. Baja eficiencia & 1 & 1,5 \\
& 2 & 5 & 7,4 \\
3 & 10 & 14,7 \\
4 & 22 & 32,4 \\
& 30 & 44,1 \\
& Total & 68 & 100,0 \\
\hline
\end{tabular}

Fuente: elaboración propia

Para llevar a cabo estas funciones, las TIC son un soporte que pueden permitir a los fabricantes optimizar los dos aspectos inmediatos a medir en las promociones en el corto plazo: la eficiencia y la eficacia. Es importante distinguir entre estos dos términos, el primero de ellos se refiere al incremento en volumen de ventas producido por una actividad promocional; el segundo, la eficacia, se refiere a las implicaciones económicas de la promoción o contribución económica.

Las TIC que mayor eficiencia y eficacia nos pueden ofrecer hoy en día son Web-Microsite y las Redes sociales por encima del correo electrónico y la telefonía móvil (tabla 4).

\section{Conclusiones}

La actividad promocional, al ser uno de los elementos de la comunicación de marca y al estar basada en el comportamiento del consumidor, se sigue considerando en la actualidad por muchas empresas como un instrumento táctico para producir un incremento de ventas en el corto plazo, sin tener en cuenta que están relacionadas con el valor de marca y que debemos englobar dentro de un proceso más complejo donde se debería relacionar con objetivos de comunicación global de la marca y su posicionamiento.

El resultado de la investigación presente ha confirmado positivamente la importancia de las Redes Sociales, y más concretamente, las Micro-sites, ofreciendo datos relevantes sobre eficiencia, mecánica promocional e implementación de la promoción. 


\section{Bibliografía}

CHANDON, P., WANSINK, B., y LAURENT, G. (2000). A benefit congruency framework of sales promotions effectiveness. Journal of Marketing. Vol. 64, octubre, pp. 65-81.

DAVIS, M., (1992). Sales Promotions as a Competitive Strategy. Management Decisión, Vol. 30, nº 7:5-10.

FERNÁNDEZ VARONA, F., LLORENTE BARROSO, C., GARCÍA GUARDIA, M.L., Madrid, (2012). Fundamentos de la Eficacia Publicitaria, Ed. Delta, Madrid.

FILL, Chris (1995). Marketing communications. Frameworks, Theories, and applications. Prentice Hall Europe.

ORTEGA MARTÍNEZ, Enrique (1989). Manual de investigación comercial, Pirámide, Madrid.

REINARES LARA, Pedro y CALVO, FERNANDEZ Sergio (1999) Gestión de la comunicación comercial; Serie MCGraw-Hill de Management.

SANTESMASES MESTRE, Miguel (1996a). Términos de Marketing. Diccionario-Base de Datos, Editorial Pirámide, Madrid.

SANTESMASES MESTRE, Miguel (1996b). Diccionario y Base de Datos de Términos de Marketing. Editorial Pirámide, Madrid.

SCHULTZ, Don E. y WALTERS, Jeffrey S. (1997). Measuring Brand Communication ROI. Associatión of National Advertisers, Inc. (ANA).

\subsection{Webgrafía}

[12-08-2013]. http://www.cob.ohio-state-edu (Fisher College of Business, Ohio University).

\section{Los autores}

Javier Sendra es Doctor por la Facultad de CC de la Información, Universidad Complutense de Madrid. MBA por el Instituto de Empresa y Licenciado en Ciencias Empresariales por la Universidad de Valencia. Actualmente es Director de Franquicias Iberia Pepsico. Directivo con más de 18 años de experiencia en empresas de Gran Consumo a nivel internacional. Destaca el libro titulado "El futuro dominio de las tecnologías de la información y comunicación en la actividad promocional".

María Tejerina Arreal profesora Facultad de Psicologia, Universidad de Murcia Profesora visitante, Psychology Department, University of Surrey, UK Research fellow Columbia University, New York, USA

María Luisa García Guardia es Doctora en Ciencias de la Información de la Universidad Complutense de Madrid, y profesora de Publicidad en dicha Universi- 
dad. Experiencia profesional en Ejecutiva de Cuentas y Directora de Cuentas. Miembro del grupo Socmedia y publicaciones en Realización Publicitaria, Diseño Digital, Técnicas de Diseño Gráfico en la Configuración de la Página Web. 